\title{
Structural underpinnings of functional plasticity in rodent visual cortex
}

\author{
C. CERRI ${ }^{1}{ }^{2}$, M. PIETRASANTA' ${ }^{1}$, L. RESTANI' \\ ${ }^{1}$ CNR Neuroscience Institute, Pisa, Italy; ${ }^{2}$ Accademia Nazionale dei Lincei, Rome, Italy
}

\begin{abstract}
A B S T R A C T
Functional plasticity in rodent visual cortex has been intensively studied since the pioneering experiments of Hubel and Wiesel in the sixties. Nevertheless, the structural modifications underlying this phenomenon remain elusive. In this article, we will review recent data focused on the dynamic of excitatory and inhibitory synapses and their structural changes linked to functional modifications. We also review novel evidence on structural remodeling that promote functional plasticity and on the role of cytoskeleton modifications in experience-dependent plasticity of rodent visual cortex.
\end{abstract}

Key words

Visual cortex $\bullet$ Spine $\bullet$ Inhibitory interneurons $\bullet$ RhoGTPase $\bullet$ Experience-dependent plasticity

\section{Introduction}

Plasticity is the neural mechanism that allows the brain to continuously adapt to changes of the external world. It sustains processes such as learning and memory and it is essential to promote recovery from brain damage. Plastic responses are particularly evident during the development of the nervous system. They have been extensively studied in the visual system, given its well-known physiology and the ease of manipulation of its primary input (Gordon and Stryker, 1996). In rodents, if one eye is sutured shut in early age (monocular deprivation, $\mathrm{MD}$ ), binocular neurons of the primary visual cortex (V1) are able to shift their eye preference (ocular dominance, OD) towards the open eye (Frenkel and Bear, 2004). Even if sensitivity of cortical circuits to MD is maximal in juvenile animals (Hofer et al., 2006; Lehmann and Lowel, 2008; Morishita and Hensch, 2008; Sato and Stryker, 2008; Spolidoro et al., 2012), the adult cortex retains some poten- tials for plasticity. Plastic changes in adulthood are qualitatively and quantitatively different from those in juvenile animals (Hofer et al., 2006; Medini and Pizzorusso, 2008). In particular, in youth MD triggers an initial depression of deprived eye inputs followed by a potentiation of the responses evoked by the open eye. Instead, in adult mice, prolonged MD results only in the potentiation of the open eye responses (Sawtell et al., 2003; Frenkel and Bear, 2004). Differently from mice, an OD shift can not be induced in rats during adulthood (Fagiolini et al., 1994; Guire et al., 1999).

Recent literature presents an increasing number of papers suggesting that functional changes due to an altered visual experience (e.g. MD, dark rearing, eyes enucleation) are often accompanied by a reorganization of neuronal structures. Excitatory synaptic structures such as dendritic spines are the most studied, as they are particularly sensitive to experience (Lendvai et al., 2000; Trachtenberg et al., 2002; Majewska and Sur, 2003; Mataga et al., 2004; 
Oray et al., 2004; Hofer et al., 2009). Recently, given the functional role of inhibition in plasticity (Hensch, 2005), several groups started to investigate the structural dynamics of inhibitory neurons both in standard conditions as well as in response to experience (Chen et al., 2011a; Chen et al., 2011b; Chen et al., 2012; van Versendaal et al., 2012).

In this review we will focus on the dynamic of excitatory and inhibitory synapses and on their structural changes related to functional modifications. We will further review novel evidence on the importance of structural remodeling to promote functional plasticity in the adult cerebral cortex (Cerri et al., 2011).

\section{Spine morphology and dynamics}

Spines are membranous protrusions from the neuronal surface. They consist of a head (volume $0.001-1 \mu \mathrm{m}^{3}$ ) connected to the neuron by a thin (diameter $<0.1 \mu \mathrm{m}$ ) spine neck (Harris and Kater, 1994; Nimchinsky et al., 2002; Zito and Murthy, 2002). They mainly arise from the dendrites but they can be also found in the soma or in the axon hillock. More than $90 \%$ of excitatory synapses terminates on spines (Yuste and Bonhoeffer, 2001; Nimchinsky et al., 2002; Sobczyk et al., 2005; Verkuyl and Matus, 2006; Tackenberg et al., 2009). Neurotransmitter receptors are mainly restricted to the surface of the spine and concentrate close to the presynaptic element. This area is referred to as the postsynaptic density (PSD), a membrane-associated disc of electron dense material, containing receptor proteins, channels, and signalling systems. In addition to PSD, the spine head contains several other specialized structures such as smooth endoplasmic reticulum (SER) and actin microfilaments, whose polymerization and de-polymerization are involved in spine morphogenesis. One important class of signalling molecules that transduce extracellular signals to regulate actin assembly is represented by the Rho family, a class of small GTPases, a subgroup of the Ras superfamily of GTPases (Hall, 1998; Lai and Ip, 2013). The best-studied members of this family are Rac1 and RhoA. Activation of Rac1 facilitates the dendritic spines formation, maintenance and elongation. In contrast, RhoA activation prevents spine formation and induces spine shortening (Luo et al., 1996; Threadgill et al., 1997; Li et al., 2000;
Nakayama and Luo, 2000; Tashiro et al., 2000; Sin et al., 2002; Govek et al., 2004).

Conventionally, spines are classified into four main categories: mushroom spines have a large head and a narrow neck; thin spines have a smaller head and a narrow neck; stubby spines show no constriction between the head and the attachment to the shaft; the filopodia, with an headless hairlike morphology, are reminiscent of the filopodium on the axonal growth cone, and both structures are found mostly during development (Skoff and Hamburger, 1974).

In the last decades, progresses in neuroscience techniques, such as the introduction of in vivo two-photon microscopy, have boosted the number of studies focused on spine structural dynamics. This imaging investigation revealed that spines are extremely dynamic structures (Bonhoeffer and Yuste, 2002). Several groups have imaged dendritic spines in the developing (Lendvai et al., 2000; Grutzendler et al., 2002; Holtmaat et al., 2005) and adult neocortex in vivo (Grutzendler et al., 2002; Trachtenberg et al., 2002; Holtmaat et al., 2005; Zuo et al., 2005; Majewska et al., 2006; Hofer et al., 2009).

The characteristics of the spine population change during the development of the mouse neocortex. Spine density increases during the second and third week of life, followed by a period of net spine pruning and loss (Holtmaat et al., 2005; Zuo et al., 2005). This leads to a fairly stable spine density in the adult brain. Young spines are often very long and classified as filopodia. Length decreases with age and in the mature brain large thick mushroom spines are frequent. Basically, large spines tend to be more stable than thin spines or filopodia (Trachtenberg et al., 2002; Holtmaat et al., 2005; Zuo et al., 2005).

During development, spines appear and disappear at a rapid rate, and as the brain matures, spine turnover decreases. In the adult brain, only a subpopulation of spines continues to turn over (Holtmaat et al., 2005; Zuo et al., 2005). These spines are transient and appear/disappear over days. All the other spines, such as the mushroom spines, are mostly stable, persisting for months (Grutzendler et al., 2002; Holtmaat et al., 2005; Zuo et al., 2005). The percentage of stable spines varies in different areas and layers of the cortex. Spines in the visual cortex are more stable than those found in the somatosensory cortex (96\% and $50 \%$ of total spines respectively) (Grutzendler et al., 2002; Trachtenberg et al., 2002; 
Holtmaat et al., 2005). In layer $2 / 3$ of the visual cortex, spines have a higher density, and there is a lower percentage of transient spines in comparison to layer 4 (Holtmaat et al., 2005).

Motility of spines decreases with age (Lendvai et al., 2000; Majewska and Sur, 2003). Some studies suggest that spine motility and the resulting changes of their morphology regulate diffusion of the spine biochemical components and regulate local calcium dynamics (Majewska et al., 2000).

In conclusion, spine morphological changes and dynamics are both hallmarks of different brain ages, with extensive remodeling in juvenile age and greater stability in adulthood.

\section{Structural dynamics of inhibitory interneurons}

Recent data revealed unexpected remarkable structural dynamics of interneurons in adult V1. Inhibitory interneurons are generally devoid of spines and receive excitatory inputs onto dendritic shafts (Kawaguchi et al., 2006; Chen et al., 2011a). While spine motility of excitatory neurons in adult $\mathrm{V} 1$ is low in normal conditions, dendritic branch tips of inhibitory interneurons display a high degree of dynamics: they can elongate or retract about $10 \mu \mathrm{m}$ over a week (Lee et al., 2006; Lee et al., 2008; Chen et al., 2011a). This remodeling process is present in various interneuron subtypes in layer $2 / 3$ (Lee et al., 2008). Interestingly, interneuron tip growth is coupled with excitatory synaptogenesis (Chen et al., 2011b). Recent evidence suggest that a percentage of interneurons expressing neuropeptide $\mathrm{Y}(\mathrm{NPY}+)$, as well as calretinin $(\mathrm{CR}+)$ and somatostatin $(\mathrm{SOM}+)$ bear spines on their dendrites (Keck et al., 2011). The turnover of the spines on these interneurons is comparable to those on pyramidal neurons (Keck et al., 2011). It is interesting that spines on interneurons carry functional glutamatergic receptors and receive excitatory inputs (Keck et al., 2011).

On the other hand, interneuron axons make contact with excitatory cells in various subcellular domains, including cell body, axon initial segment, dendritic shaft, and also dendritic spines (Markram et al., 2004). Imaging studies revealed a certain degree of stability in the overall axonal architecture of inhibitory neurons during normal experience (Marik et al.,
2010; Keck et al., 2011). However, their axonal boutons are constantly added and lost over time (Chen et al., 2011b; Keck et al., 2011).

An interesting in vivo experiment (by high-resolution dual-colour two-photon microscopy) simultaneously monitored inhibitory synapses and dendritic spines in the entire dendritic arbor of layer $2 / 3$ pyramidal neurons (Chen et al., 2012). The results revealed the distribution and the structural dynamics of inhibitory synapses on both dendritic shafts and spines of postsynaptic pyramidal neurons. Authors took advantage of fluorescent tagging of gephiryn, a postsynaptic protein found exclusively in inhibitory synapses, to image inhibitory structures (Chen et al., 2012; van Versendaal et al., 2012). By this new technique, they demonstrated that inhibitory synapses on dendritic shaft are uniformly distributed across the dendritic tree. On the other hand, it was clear that inhibitory synapses on spines were at high density on distal apical dendrites of the pyramidal neuron (Chen et al., 2012). It was also possible to measure turnover rate of inhibitory synapses on spines that was about threefold higher than that of inhibitory synapses on shafts (Chen et al., 2012). Interestingly, inhibitory synapses and dendritic spines dynamics are spatially clustered indicating local coordination of inhibitory and excitatory synaptic rearrangements (Chen et al., 2012).

\section{Structural changes and sensory experience}

The idea that changes in the structure of the brain might occur as a consequence of experience was first proposed by Ramon y Cajal more than a century ago. Many subsequent studies have confirmed this predictive idea. Indeed, changes in spine density, morphology and motility have been shown to occur with changes in neuronal activity and experience. Engert and Bonhoeffer (1999) provided a bright demonstration of this hypothesis. Combining the stimulation pattern of long term potentiation (LTP) induction with two-photon imaging in hippocampal slice culture, they found that the induction of LTP in a small dendritic region resulted in the emergence of new spines in the stimulated region. Thus, the strengthening of neuronal connections is sustained by structural changes in dendrites (Engert and Bonhoeffer, 1999). Similar effects on spines were 
described also after sensory stimulation. Indeed, raising rats in an enriched environment (Diamond et al., 1975; Leggio et al., 2005) or whisker deflection in freely moving animals (Knott et al., 2002) lead to an increase in spine and synapse densities.

Many of the studies focused on spines plasticity have been carried out in somatosensory cortex of adult mouse as a model. Here the spine population appears to be much more dynamic than in other cortical areas (Trachtenberg et al., 2002; Holtmaat et al., 2006). Wilbrecht and co-authors investigated responses to whisker deflection in the barrel cortex (Wilbrecht et al., 2010). They trimmed a subset of whiskers and evaluated the responses evoked by the spared whiskers. Authors found that the response to spared whiskers was potentiated. In particular, they recorded a marked potentiation in layer 5 neurons close to the interface between deprived and spared cortical barrel columns. In this region, trimming induced growth of new, persistent spines. Interestingly, in homozygote aCAMKII-T286A mice, that lack experience-dependent potentiation, whisker trimming resulted in the generation but not stabilization of new spines (Wilbrecht et al., 2010). Overall, these findings provide strong support for a role of spine growth and stabilization in response to potentiation. This is in line with more recent data demonstrating a link between spine density and functional potentiation in visual cortex (Hofer et al., 2009; Cerri et al., 2011, see below).

Evidence for a role of spine growth in experiencedependent plasticity also came from experiments in the mouse visual cortex. In juvenile animals, functional changes after MD come first, and thalamocortical axon remodeling takes some days to develop (Antonini and Stryker, 1993; Antonini et al., 1999). Spine motility increases after 2 days of $\mathrm{MD}$, while after 4 days there is a significant spine loss, consistent with the initial strong reduction of responses elicited by the deprived eye (Gordon and Stryker, 1996; Mataga et al., 2004; Oray et al., 2004). An additional period of MD in juvenile mice lead to the formation of new spines, in keeping with the subsequent strengthening of open eye inputs (Mataga et al., 2004). Similarly, a study by Sur and collaborators, combining two-photon microscopy and intrinsic signal imaging, found that spines loss closely follows functional depression of deprived eye input in ferret visual cortex (Yu et al., 2011).
In contrast, in the adult mouse, the MD-induced OD shift is implemented primarily by the strengthening of the open eye responses (Sawtell et al., 2003). Monocular deprivation doubles the rate of spine formation, thereby increasing spine density of layer 5 neurons of the binocular visual cortex (Hofer et al., 2009). New spine formation during MD seems to be strictly correlated with the potentiation of the responses evoked by the open eye.

Interestingly, spine growth is specific to layer 5 cells, as in the somatosensory cortex, suggesting a circuit-specific structural plasticity. Moreover, spine loss after MD is not observed in adulthood, consistent with the absence of depression of deprived eye inputs (Sawtell et al., 2003; Mataga et al., 2004).

A nice paper by Sonja Hofer and co-authors provided interesting results on structural plasticity and MD in adulthood (Hofer et al., 2009). They followed spine dynamics of layer 5 neurons in the adult binocular visual cortex during ocular dominance plasticity. They performed a first MD, then mice were allowed normal binocular vision for about 16-20 days, and after they repeated a second MD. The first MD induced the expected OD shift, and was paralleled by a doubling in rate of spine formation, that increased spine density. Restoring binocular vision returned spine dynamics to baseline levels, but, in contrast, absolute spine density remained elevated. However, when the same eye was deprived for the second time, despite the observed OD shift, spines did not increase again. These results suggest that the synapses on the spines generated during the first MD could be weakened by binocular vision to restore a normal ocular dominance, and strengthened by the second $\mathrm{MD}$, providing the structural base for the faster OD shift induced by the second MD.

These beautiful results propose a model that could explain how prior experiences could be stored in adult cortical circuits (Hofer et al., 2009).

Taken together, these results demonstrate that gain or loss of spines during MD closely parallels the functional consequences of MD and corroborates the idea of a causal link between experience-dependent plasticity and structural changes.

Other paradigms of sensory deprivation have also linked spine reorganization with functional outcomes. A total lack of visual experience in the early stage of life (Dark Rearing) dampens visual responses and shifts the feature of spine morphology 
and density towards their immature state that can only be partially reverted by light exposure (Wallace and Bear, 2004; Tropea et al., 2010). A small retinal lesion, but not total lack of visual input, highly increases spine turnover in visual cortex, leading to an almost complete replacement of spines in the deafferentated cortex. This process is likely to mediate the topographic remapping linked with visual function recovery (Keck et al., 2008).

\section{The role of MATRIX in spine dynamics}

In the last decade, it has became clear that the extracellular environment, such as the Extracellular Matrix (ECM), can play a crucial role in spine dynamics and visual cortical plasticity (Pizzorusso et al., 2002; Pizzorusso et al., 2006; Spolidoro et al., 2012; de Vivo et al., 2013). Indeed, several evidences suggest that in adults, ECM is strongly non-permissive for spine plasticity.

The family of chondroitin-sulfate proteoglycans (CSPGs) is one of the major components of the ECM that have been found to be involved in plastic remodeling. These molecules are organized in specific structures named perineuronal nets (PNNs) that lay around the soma and dendrites of parvalbumin-positive neurons. Degradation of CSPGs in adult rats visual cortex by chondroitinase $\mathrm{ABC}$ (ChABC) leads to restoration of OD plasticity (normally absent in adult rats) and to an increase spine density (Pizzorusso et al., 2002; Pizzorusso et al., 2006). Recently, two-photon in vivo spine imaging in mice, showed an increased structural plasticity of spines (i.e. higher spine motility and appearance of filopodia) upon degradation of CSPGs (de Vivo et al., 2013). Moreover, ChABC treatment followed by high frequency stimulation induces LTP in layer 5 neurons of the visual cortex and results in spines head enlargement (de Vivo et al., 2013).

\section{Experience-dependent dynamics of inter- neurons}

Recent papers have addressed the structural dynamics of inhibitory neurons in response to alteration of visual inputs (Keck et al., 2011; Chen et al., 2012; van Versendaal et al., 2012). Keck and colleagues showed that the retinal lesion paradigm induces a general decrease in axonal boutons density in the majority of inhibitory neurons, and a specific, rapid and long lasting reduction of spines in spiny interneurons (Keck et al., 2011). Interestingly, authors observed that these changes occurred hours after retinal lesion, suggesting that inhibitory structural plasticity is likely to precede changes in excitatory circuits.

Other works took advantage of the MD paradigm to trigger structural changes in mice (Chen et al., 2011b; Chen et al., 2012; van Versendaal et al., 2012). MD increases branch dynamics of interneurons, likely providing the substrate for the reduction of intracortical inhibition that allows plasticity to occur (Chen et al., 2011b).

MD determines, already within the first day, a transient loss of inhibitory synapses on pyramidal spines imaged in layer $2 / 3$ of the visual cortex in adult mice. Surprisingly, restoration of binocular vision is again associated with inhibitory synapse loss, and increases the responsiveness to the previously deprived eye (van Versendaal et al., 2012). These mechanisms may represent an important way to modulate specific inputs, without interfering with excitatory structure dynamics and preserving the general organization of the circuitry.

In line with these findings, Chen and co-authors also reported a reduction in inhibitory synapses on spines within 2 days after MD, at an early time with respect to inhibitory synapses on dendritic shaft, eliminated between 4-8 days after MD (Chen et al., 2012). These data suggest that inhibitory synapses on shaft and on spines constitute two populations with different kinetics, likely to be differentially affected by experience (Chen et al., 2012).

All the studies described above provide a link between structural and functional plasticity as they consider changes of neuronal structures that follow the alteration of neuronal activity or sensory experience. Another approach to study the relationship between structure and function is to experimentally induce structural changes first, and then look at the functional effects on neuronal circuits (Dahlhaus et al., 2008; Cerri et al., 2011; Djurisic et al., 2013). For example, reducing dendritic spines and filopodia density limits LTP induction in those connections arising from layer 4 to layer 2/3 of mouse visual cortex (Dahlhaus et al., 2008).

Our group proposed a direct link between spine dynamic and adult plasticity. We performed an experiment that proved that pharmacological enhancement of spine density resulted in reinstatement of OD plasticity in adult rats (Cerri et al., 2011). 


\section{Structural remodeling via RhoGTPases activation triggers functional plasticity}

As mentioned before, actin dynamics is a key regulator of the shape and morphology of plastic axons, dendrites, and dendritic spines. Actin dynamics is controlled by a family of small GTPases, the Rho family which include RhoA, Rac1, and Cdc42 (Van Aelst and Cline, 2004). Rho GTPases are molecular switches that cycle between a GDP-bound inactive and a GTP-bound active state to control actin assembly and polymerization. They are involved in many neuronal development processes that require morphological changes, like neuronal migration and axon growth and guidance.

The importance of Rho GTPases for morphogenesis and maturation of dendritic spine and branches has been indirectly demonstrated by modulating the expression of Rho pathway regulators (Penzes et al., 2003; Buttery et al., 2006; Kang et al., 2009; Impey et al., 2010). For example, increased expression of $\alpha 1$-chimaerin, a RhoGAP that inactivates Rac, results in the pruning of dendritic spines and branches. By contrast, suppression of its expression resulted in increased process growth from the dendritic shaft (Buttery et al., 2006).

Recently, we exploited cytotoxic necrotizing factor 1 (CNF1), a bacterial toxin from Escherichia coli able to block Rho GTPases in their activated, GTP-bound state, to study whether stimulation of structural rearrangements reinstates experiencedependent plasticity in adult rats (Cerri et al., 2011). CNF1 induces deamidation of a single glutamine residue of the Rho molecules, thus impeding GTP hydrolysis and leading to their persistent activation (Fiorentini et al., 1997; Flatau et al., 1997; Schmidt et al., 1997; Diana et al., 2007; Fabbri et al., 2010). We unilaterally injected CNF1 into the primary visual cortex of adult rats and we observed a longlasting activation of Rac1 (10 days). To evaluate CNF1 impact on spines structure, we performed "DiOlistic" labeling of pyramidal neurons in layers $2 / 3$ of the primary visual cortex, 10 days after the toxin injection. We found that the median length of the spine neck in CNF1-treated cortices was significantly higher than in control cortices. Moreover, we detected an increase in spine density of CNF1treated animals (Fig. 1a,b,c). Spine analysis revealed that the vast majority of these protusions had a well-defined neck and head structure, characteristic of mature spines (Fig. 1a). This is consistent with recent data obtained by Martino and co-workers that show an increase in spine density in basal but not apical dendrites in V1, following CNF1 intracerebroventricular delivery (Martino et al., 2013).

Enhanced spine density in CNF1 treated animals was also coupled with a corresponding increase in presynaptic excitatory markers, as shown by increased levels of vGlut1 (the major excitatory vesicular transporter in the cortex) and vGlut2 (a marker for thalamocortical axon terminals) (Fig. 1d,e). These data showed an increased density of intracortical and geniculocortical afferents in treated rats.

To address whether CNF1-induced structural remodeling reinstates functional plasticity, adult animals were monocularly deprived for 7 days, contralateral to the CNF-1 injection performed 7 days before MD. Delivery of CNF1 prior to MD resulted in significant OD shift towards the open eye, as shown both by Visual Evoked Potentials (VEPs) and extracellular spiking activity (Fig. 1f,g,h). Importantly, the OD shift was selectively due to a significant increase in the strength of inputs from the open eye. The enhancement in geniculocortical connectivity, shown by vGLUT2 levels, is in line with the potentiation of the open-eye responses, suggesting the addition of synaptic terminals serving the ipsilateral, nondeprived eye in the CNF1-treated cortex.

Interestingly, CNF1 produces no changes in the expression of known structural "brakes" on adult plasticity, such as myelin and perineuronal nets. Therefore, the reactivation of OD plasticity by CNF1 appears to involve morphological changes that proceed independently of these well-described brakes on plasticity. Thus, these results provide good evidence on the importance of cytoskeleton changes in inducing functional plasticity in the adult cerebral cortex.

We speculate that the increase in the density of spines could be a crucial factor for the reactivation of plasticity. This suggests an increase in the number of available postsynaptic sites that could trigger the wiring up of new synapses. CNF1 sets in motion a mechanism of activity-dependent takeover by which newly formed dendritic sites may be contacted preferentially by more active afferents from the open eye. These results indicate a direct link between structural and functionalplasticity of the adult brain and demonstrate a key role for Rho GTPases in these processes. 


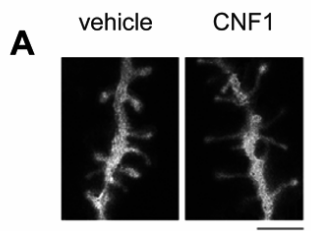

C

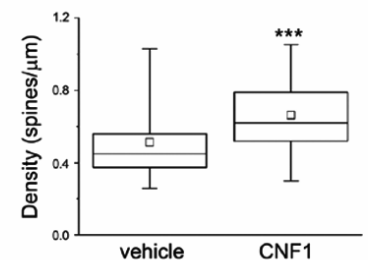

D

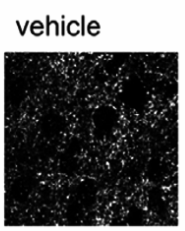

CNF1

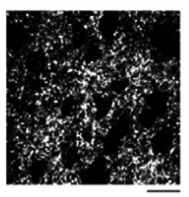

E

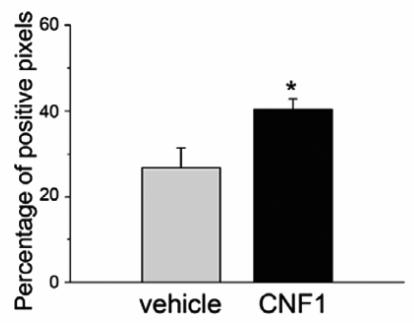

$\mathbf{F}$

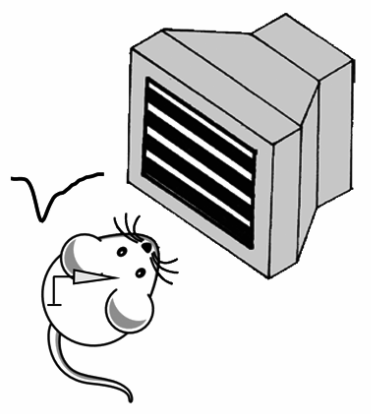

G

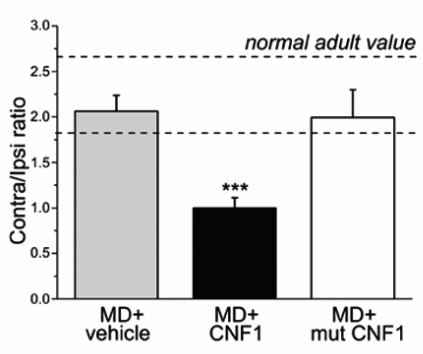

H

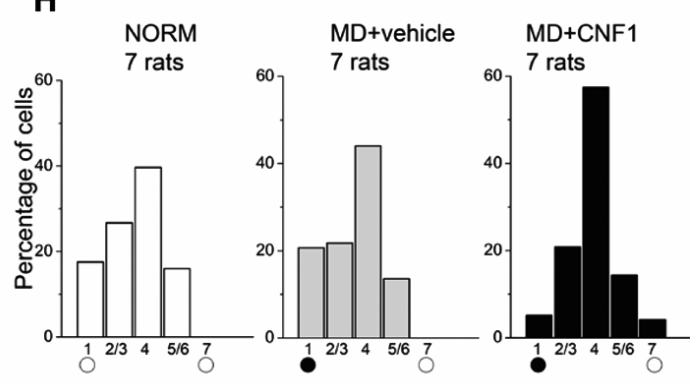

Fig. 1. - Structural remodeling via Rho GTPases activation triggers functional plasticity. a-b-c-d-e) Increased structural remodeling in the primary visual cortex after CNF1 treatment.

a) Spine phenotype of visual cortex pyramidal neurons in animals treated with vehicle (left) or CNF1 (right). Scale bar, $1 \mu \mathrm{m}$. b) Box chart showing spine neck lengths for vehicle and CNF1-treated neurons. Spine neck length is significantly longer in CNF1-treated samples with respect to controls (Mann-Whitney rank-sum test, p < 0.001 ). c) Analysis of spine density was performed on basal dendrites from layer 2/3 pyramidal neurons in visual cortex. Neurons of animals treated with CNFl show a consistent and highly significant increase in the density of spines (Mann-Whitney rank-sum test, $\mathrm{p}<0.001$ ). d) Immunostaining for vGlut-2 in layer 4 of binocular visual cortex in vehicle-and CNF1-treated rats. Scale bar, $10 \mu \mathrm{m}$. e) Quantification reveals a significant increase in the density of vGlut-2-positive puncta in the CNF1-infused cortex ( $\dagger$ test, $p=0.04$ ). These data suggest an increased density of geniculocortical afferents in CNF1-treated cortex. $\mathrm{f}-\mathrm{g}-\mathrm{h}$ ) Activation of Rho GTPases reinstates OD plasticity in the adult cortex. f) Experimental setup for in vivo testing of OD plasticity by electrophysiological recordings g) C/I VEP ratios in MD adult rats treated with vehicle (MD + vehicle), with CNF1 (MD + CNF1) and with a mutated form of CNF1 (MD + mut CNF1). In MD + vehicle rats, the C/I ratio is unchanged (post-ANOVA Holm-Sidak test, $\mathrm{p}=0.52$ ) compared with the normal adult range (indicated by the dashed lines), whereas in MD + CNF1 animals, there is a dramatic decrease of the $\mathrm{C} / \mathrm{I}$ ratio $(\mathrm{p}<0.001$ ). Injection of a mutated form of CNF1 (mut CNF1) is completely ineffective in shifting $\mathrm{OD}(\mathrm{MD}+$ mut CNF1 vs. $\mathrm{MD}+$ vehicle, $\mathrm{p}=0.8 ; \mathrm{MD}+$ mut CNF1 vs. $\mathrm{MD}+\mathrm{CNF1}, \mathrm{p}=0.002)$. Normal adult range: mean \pm SD value. e) OD distributions of naive adult rats (NORM) and rats monocularly deprived for 7 days and treated with either vehicle (MD + vehicle) or CNF1 (MD + CNF1). Recordings were performed in the visual cortex contralateral to the closed eye (filled circle). CNF1 delivery prior to MD triggers a significant OD shift toward the open eye and a reduction of the proportion of neurons driven exclusively by the closed eye $\left(\chi^{2}\right.$ test, $\left.p<0.001\right)$. Data are mean \pm SEM (modified from Cerri et al., 2011).

\section{Summary}

Here we review important findings in the field of structural changes that underlie functional plasticity with particular attention to the visual cortex. We focus on the importance of data that describe spine remodeling in excitatory neurons and the recent discovery of the remarkable structural dynamics of interneurons in adult V1. Recent progresses in the field of Neuroscience with the help of innovative microscopy techniques, allow a deep characterisation of neuronal structures and the description 
of their live changes. We address how structural remodeling parallels functional modifications in experience-dependent plasticity, commenting on the most relevant findings on this topic. All reported data suggest the existence of a close link between structural and functional plasticity. In particular, our data demonstrate that pharmacological enhancement of spine density and length resulted in reinstatement of OD plasticity in adult rats. These results point out that structural remodeling strictly contributes to experience-dependent plasticity and they represent the first demonstration of a causal link between structural and functional plasticity.

Taken together the experiments described here emphasize the key role of neuronal structural changes in the evergrowing field of visual cortical physiology.

\section{Acknowledgements}

$\mathrm{CC}$ is supported by the Accademia Nazionale dei Lincei (Rome) and LR is supported by Italian Ministry of Research (FIRB2010-RBFR10ZBYZ_003).

\section{References}

Antonini A., Fagiolini M., Stryker M.P. Anatomical correlates of functional plasticity in mouse visual cortex. J. Neurosci., 19: 4388-4406, 1999.

Antonini A. and Stryker M.P. Rapid remodeling of axonal arbors in the visual cortex. Science, 260: 1819-1821, 1993.

Bonhoeffer T. and Yuste R. Spine motility. Phenomenology, mechanisms, and function. Neuron, 35: 1019-1027, 2002.

Buttery P., Beg A.A., Chih B., Broder A., Mason C.A., Scheiffele P. The diacylglycerol-binding protein alpha1-chimaerin regulates dendritic morphology. Proc. Natl. Acad. Sci. USA, 103: 19241929, 2006.

Cerri C., Fabbri A., Vannini E., Spolidoro M., Costa M., Maffei L., Fiorentini C., Caleo M. Activation of Rho GTPases triggers structural remodeling and functional plasticity in the adult rat visual cortex. J. Neurosci., 31: 15163-15172, 2011.

Chen J.L., Flanders G.H., Lee W.C., Lin W.C., Nedivi E. Inhibitory dendrite dynamics as a general feature of the adult cortical microcircuit. $J$. Neurosci., 31: 12437-12443, 2011 a.

Chen J.L., Lin W.C., Cha J.W., So P.T., Kubota Y., Nedivi E. Structural basis for the role of inhibition in facilitating adult brain plasticity. Nat. Neurosci., 14: 587-594, $2011 \mathrm{~b}$.

Chen J.L., Villa K.L., Cha J.W., So P.T., Kubota Y., Nedivi E. Clustered dynamics of inhibitory synapses and dendritic spines in the adult neocortex. Neuron, 74: 361-373, 2012.

Dahlhaus M., Hermans J.M., Van Woerden L.H., Saiepour M.H., Nakazawa K., Mansvelder H.D., Heimel J.A., Levelt C.N. Notch1 signaling in pyramidal neurons regulates synaptic connectivity and experience-dependent modifications of acuity in the visual cortex. J. Neurosci., 28: 10794-10802, 2008.

de Vivo L., Landi S., Panniello M., Baroncelli L., Chierzi S., Mariotti L., Spolidoro M., Pizzorusso T., Maffei L., Ratto G.M. Extracellular matrix inhibits structural and functional plasticity of dendritic spines in the adult visual cortex. Nat. Commun., 4: 1484, 2013.

Diamond M.C., Lindner B., Johnson R., Bennett E.L., Rosenzweig M.R. Differences in occipital cortical synapses from environmentally enriched, impoverished, and standard colony rats. J. Neurosci. Res., 1: 109-119, 1975.

Diana G., Valentini G., Travaglione S., Falzano L., Pieri M., Zona C., Meschini S., Fabbri A., Fiorentini C. Enhancement of learning and memory after activation of cerebral Rho GTPases. Proc. Natl. Acad. Sci. USA, 104: 636-641, 2007.

Djurisic M., Vidal G.S., Mann M., Aharon A., Kim T., Ferrao Santos A., Zuo Y., Hubener M., Shatz C.J. PirB regulates a structural substrate for cortical plasticity. Proc. Natl. Acad. Sci. USA, 110: 20771-20776, 2013.

Engert F. and Bonhoeffer T. Dendritic spine changes associated with hippocampal long-term synaptic plasticity. Nature, 399: 66-70, 1999.

Fabbri A., Travaglione S., Fiorentini C. Escherichia coli cytotoxic necrotizing factor 1 (CNF1): toxin biology, in vivo applications and therapeutic potential. Toxins (Basel), 2: 283-296, 2010.

Fagiolini M., Pizzorusso T., Berardi N., Domenici L., Maffei L. Functional postnatal development of the rat primary visual cortex and the role of visual experience: dark rearing and monocular deprivation. Vision Res., 34: 709-720, 1994.

Fiorentini C., Fabbri A., Flatau G., Donelli G., Matarrese P., Lemichez E., Falzano L., Boquet P. Escherichia coli cytotoxic necrotizing factor 1 (CNF1), a toxin that activates the Rho GTPase. $J$. Biol. Chem., 272: 19532-19537, 1997.

Flatau G., Lemichez E., Gauthier M., Chardin P., Paris S., Fiorentini C., Boquet P. Toxin-induced 
activation of the $\mathrm{G}$ protein $\mathrm{p} 21$ Rho by deamidation of glutamine. Nature, 387: 729-733, 1997.

Frenkel M.Y. and Bear M.F. How monocular deprivation shifts ocular dominance in visual cortex of young mice. Neuron, 44: 917-923, 2004.

Gordon J.A. and Stryker M.P. Experience-dependent plasticity of binocular responses in the primary visual cortex of the mouse. J. Neurosci., 16: 32743286, 1996.

Govek E.E., Newey S.E., Akerman C.J., Cross J.R., Van der Veken L., Van Aelst L. The X-linked mental retardation protein oligophrenin-1 is required for dendritic spine morphogenesis. Nat. Neurosci., 7: 364-372, 2004.

Grutzendler J., Kasthuri N., Gan W.B. Long-term dendritic spine stability in the adult cortex. Nature, 420: 812-816, 2002.

Guire E.S., Lickey M.E., Gordon B. Critical period for the monocular deprivation effect in rats: assessment with sweep visually evoked potentials. $J$. Neurophysiol., 81: 121-128, 1999.

Hall A. Rho GTPases and the actin cytoskeleton. Science, 279: 509-514, 1998.

Harris K.M., Kater S.B. Dendritic spines: cellular specializations imparting both stability and flexibility to synaptic function. Annu. Rev. Neurosci., 17: 341-371, 1994.

Hensch T.K. Critical period mechanisms in developing visual cortex. Curr. Top. Dev. Biol., 69: 215237, 2005.

Hofer S.B., Mrsic-Flogel T.D., Bonhoeffer T., Hubener M. Lifelong learning: ocular dominance plasticity in mouse visual cortex. Curr. Opin. Neurobiol., 16: 451-459, 2006.

Hofer S.B., Mrsic-Flogel T.D., Bonhoeffer T., Hubener M. Experience leaves a lasting structural trace in cortical circuits. Nature, 457: 313-317, 2009.

Holtmaat A., Wilbrecht L., Knott G.W., Welker E., Svoboda K. Experience-dependent and cell-typespecific spine growth in the neocortex. Nature, 441: 979-983, 2006.

Holtmaat A.J., Trachtenberg J.T., Wilbrecht L., Shepherd G.M., Zhang X., Knott G.W., Svoboda $\mathrm{K}$. Transient and persistent dendritic spines in the neocortex in vivo. Neuron, 45: 279-291, 2005.

Impey S., Davare M., Lesiak A., Fortin D., Ando H., Varlamova O., Obrietan K., Soderling T.R., Goodman R.H., Wayman G.A. An activityinduced microRNA controls dendritic spine formation by regulating Rac1-PAK signaling. Mol. Cell. Neurosci., 43: 146-156, 2010.
Kang M.G., Guo Y., Huganir R.L. AMPA receptor and GEF-H1/Lfc complex regulates dendritic spine development through RhoA signaling cascade. Proc. Natl. Acad. Sci. USA, 106: 3549-3554, 2009.

Kawaguchi Y., Karube F., Kubota Y. Dendritic branch typing and spine expression patterns in cortical nonpyramidal cells. Cereb. Cortex, 16: 696-711, 2006.

Keck T., Mrsic-Flogel T.D., Vaz Afonso M., Eysel U.T., Bonhoeffer T., Hubener M. Massive restructuring of neuronal circuits during functional reorganization of adult visual cortex. Nat. Neurosci., 11: 1162-1167, 2008.

Keck T., Scheuss V., Jacobsen R.I., Wierenga C.J., Eysel U.T., Bonhoeffer T., Hubener M. Loss of sensory input causes rapid structural changes of inhibitory neurons in adult mouse visual cortex. Neuron, 71: 869-882, 2011.

Knott G.W., Quairiaux C., Genoud C., Welker E. Formation of dendritic spines with GABAergic synapses induced by whisker stimulation in adult mice. Neuron, 34: 265-273, 2002.

Lai K.O. and Ip N.Y. Structural plasticity of dendritic spines: the underlying mechanisms and its dysregulation in brain disorders. Biochim. Biophys. Acta, 1832: 2257-2263, 2013.

Lee W.C., Chen J.L., Huang H., Leslie J.H., Amitai Y., So P.T., Nedivi E. A dynamic zone defines interneuron remodeling in the adult neocortex. Proc. Natl. Acad. Sci. USA, 105: 19968-19973, 2008.

Lee W.C., Huang H., Feng G., Sanes J.R., Brown E.N., So P.T., Nedivi E. Dynamic remodeling of dendritic arbors in GABAergic interneurons of adult visual cortex. PLoS Biol., 4: e29, 2006.

Leggio M.G., Mandolesi L., Federico F., Spirito F., Ricci B., Gelfo F., Petrosini L. Environmental enrichment promotes improved spatial abilities and enhanced dendritic growth in the rat. Behav. Brain Res., 163: 78-90, 2005.

Lehmann K. and Lowel S. Age-dependent ocular dominance plasticity in adult mice. PLoS One, 3: e3120, 2008.

Lendvai B., Stern E.A., Chen B., Svoboda K. Experience-dependent plasticity of dendritic spines in the developing rat barrel cortex in vivo. Nature, 404: 876-881, 2000.

Li Z., Van Aelst L., Cline H.T. Rho GTPases regulate distinct aspects of dendritic arbor growth in Xenopus central neurons in vivo. Nat. Neurosci., 3: 217-225, 2000. 
Luo L., Hensch T.K., Ackerman L., Barbel S., Jan L.Y., Jan Y.N. Differential effects of the Rac GTPase on Purkinje cell axons and dendritic trunks and spines. Nature, 379: 837-840, 1996.

Majewska A. and Sur M. Motility of dendritic spines in visual cortex in vivo: changes during the critical period and effects of visual deprivation. Proc. Natl. Acad. Sci. USA, 100: 16024-16029, 2003.

Majewska A., Tashiro A., Yuste R. Regulation of spine calcium dynamics by rapid spine motility. $J$. Neurosci., 20: 8262-8268, 2000.

Majewska A.K., Newton J.R., Sur M. Remodeling of synaptic structure in sensory cortical areas in vivo. J. Neurosci., 26: 3021-3029, 2006.

Marik S.A., Yamahachi H., McManus J.N., Szabo G., Gilbert C.D. Axonal dynamics of excitatory and inhibitory neurons in somatosensory cortex. PLoS Biol., 8: e1000395, 2010.

Markram H., Toledo-Rodriguez M., Wang Y., Gupta A., Silberberg G., Wu C. Interneurons of the neocortical inhibitory system. Nat. Rev. Neurosci., 5: 793-807, 2004.

Martino A., Ettorre M., Musilli M., Lorenzetto E., Buffelli M., Diana G. Rho GTPase-dependent plasticity of dendritic spines in the adult brain. Front. Cell. Neurosci., 7: 62, 2013.

Mataga N., Mizuguchi Y., Hensch T.K. Experiencedependent pruning of dendritic spines in visual cortex by tissue plasminogen activator. Neuron, 44: 1031-1041, 2004.

Medini P. and Pizzorusso T. Visual experience and plasticity of the visual cortex: a role for epigenetic mechanisms. Front. Biosci., 13: 3000-3007, 2008.

Morishita H. and Hensch T.K. Critical period revisited: impact on vision. Curr. Opin. Neurobiol., 18: 101-107, 2008.

Nakayama A.Y. and Luo L. Intracellular signaling pathways that regulate dendritic spine morphogenesis. Hippocampus, 10: 582-586, 2000.

Nimchinsky E.A., Sabatini B.L., Svoboda K. Structure and function of dendritic spines. Annu. Rev. Physiol., 64: 313-353, 2002.

Oray S., Majewska A., Sur M. Dendritic spine dynamics are regulated by monocular deprivation and extracellular matrix degradation. Neuron, 44: 1021-1030, 2004.

Penzes P., Beeser A., Chernoff J., Schiller M.R., Eipper B.A., Mains R.E., Huganir R.L. Rapid induction of dendritic spine morphogenesis by trans-synaptic ephrinB-EphB receptor activation of the Rho-GEF kalirin. Neuron, 37: 263-274, 2003.
Pizzorusso T., Medini P., Berardi N., Chierzi S., Fawcett J.W., Maffei L. Reactivation of ocular dominance plasticity in the adult visual cortex. Science, 298: 1248-1251, 2002.

Pizzorusso T., Medini P., Landi S., Baldini S., Berardi N., Maffei L. Structural and functional recovery from early monocular deprivation in adult rats. Proc. Natl. Acad. Sci. USA, 103: 85178522, 2006.

Sato M. and Stryker M.P. Distinctive features of adult ocular dominance plasticity. J. Neurosci., 28: 10278-10286, 2008.

Sawtell N.B., Frenkel M.Y., Philpot B.D., Nakazawa K., Tonegawa S., Bear M.F. NMDA receptordependent ocular dominance plasticity in adult visual cortex. Neuron, 38: 977-985, 2003.

Schmidt G., Sehr P., Wilm M., Selzer J., Mann M., Aktories K. Gln 63 of Rho is deamidated by Escherichia coli cytotoxic necrotizing factor-1. Nature, 387: 725-729, 1997.

Sin W.C., Haas K., Ruthazer E.S., Cline H.T. Dendrite growth increased by visual activity requires NMDA receptor and Rho GTPases. Nature, 419: 475-480, 2002.

Skoff R.P. and Hamburger V. Fine structure of dendritic and axonal growth cones in embryonic chick spinal cord. J. Comp. Neurol., 153: 107-147, 1974.

Sobczyk A., Scheuss V., Svoboda K. NMDA receptor subunit-dependent $[\mathrm{Ca} 2+]$ signaling in individual hippocampal dendritic spines. J. Neurosci., 25: 6037-6046, 2005.

Spolidoro M., Putignano E., Munafo C., Maffei L., Pizzorusso T. Inhibition of matrix metalloproteinases prevents the potentiation of nondeprived-eye responses after monocular deprivation in juvenile rats. Cereb. Cortex, 22: 725-734, 2012.

Tackenberg C., Ghori A., Brandt R. Thin, stubby or mushroom: spine pathology in Alzheimer's disease. Curr. Alzheimer Res., 6: 261-268, 2009.

Tashiro A., Minden A., Yuste R. Regulation of dendritic spine morphology by the rho family of small GTPases: antagonistic roles of Rac and Rho. Cereb. Cortex, 10: 927-938, 2000.

Threadgill R., Bobb K., Ghosh A. Regulation of dendritic growth and remodeling by Rho, Rac, and Cdc42. Neuron, 19: 625-634, 1997.

Trachtenberg J.T., Chen B.E., Knott G.W., Feng G., Sanes J.R., Welker E., Svoboda K. Long-term in vivo imaging of experience-dependent synaptic plasticity in adult cortex. Nature, 420: 788-794, 2002. 
Tropea D., Majewska A.K., Garcia R., Sur M. Structural dynamics of synapses in vivo correlate with functional changes during experience-dependent plasticity in visual cortex. J. Neurosci., 30: 11086-11095, 2010.

Van Aelst L. and Cline H.T. Rho GTPases and activity-dependent dendrite development. Curr. Opin. Neurobiol., 14: 297-304, 2004.

van Versendaal D., Rajendran R., Saiepour M.H., Klooster J., Smit-Rigter L., Sommeijer J.P., De Zeeuw C.I., Hofer S.B., Heimel J.A., Levelt C.N. Elimination of inhibitory synapses is a major component of adult ocular dominance plasticity. Neuron, 74: 374-383, 2012.

Verkuyl J.M. and Matus A. Time-lapse imaging of dendritic spines in vitro. Nat. Protoc., 1: 23992405, 2006.

Wallace W. and Bear M.F. A morphological correlate of synaptic scaling in visual cortex. J. Neurosci., 24: 6928-6938, 2004.
Wilbrecht L., Holtmaat A., Wright N., Fox K., Svoboda K. Structural plasticity underlies experience-dependent functional plasticity of cortical circuits. J. Neurosci., 30: 4927-4932, 2010.

Yu H., Majewska A.K., Sur M. Rapid experiencedependent plasticity of synapse function and structure in ferret visual cortex in vivo. Proc. Natl. Acad. Sci. USA, 108: 21235-21240, 2011.

Yuste R. and Bonhoeffer T. Morphological changes in dendritic spines associated with long-term synaptic plasticity. Annu. Rev. Neurosci., 24: 10711089, 2001.

Zito K. and Murthy V.N. Dendritic spines. Curr. Biol., 12: R5, 2002.

Zuo Y., Lin A., Chang P., Gan W.B. Development of long-term dendritic spine stability in diverse regions of cerebral cortex. Neuron, 46: 181-189, 2005 . 